\title{
Le jeune chercheur et l'interdisciplinarité en sciences sociales
}

\section{Des pratiques remises en question}

\author{
Ève Anne Bühler ${ }^{\mathrm{a}}$, Fabienne Cavailléb ${ }^{\mathrm{b}}$, Mélanie Gambino ${ }^{\mathrm{c}}$ \\ a Doctorante en études rurales, UMR Dynamiques rurales, ENSAT, avenue de l'Agrobiopole, BP 32607, \\ 31326 Castanet Tolosan cedex, France \\ b Maître de conférences en géographie, UMR Dynamiques rurales, Pavillon de la recherche, 5 allée Antonio Machado, \\ 31058 Toulouse cedex 9, France \\ c Doctorante en géographie, UMR Dynamiques rurales, Pavillon de la recherche, 5 allée Antonio Machado, \\ 31058 Toulouse cedex 9, France
}

\section{Mots-clés :} interdisciplinarité; jeune chercheur ; épistémologie ; légitimité scientifique
Résumé - La pratique individuelle de l'interdisciplinarité par de jeunes chercheurs est rarement questionnée. Elle est pourtant bien spécifique. L'emprunt de concepts, de méthodes, de théories aux autres disciplines s'effectue souvent d'abord de manière intuitive, sur le mode de l'affinité. Il est donc important pour le jeune chercheur de réfléchir aux exigences de la scientificité dans le cadre original de l'interdisciplinarité. La difficulté est en effet de parvenir à une cohérence épistémologique et, pour ce faire, d'acquérir une «polycompétence» indispensable. L'interdisciplinarité est en fait plurielle. Deux modalités de l'interdisciplinarité peuvent être différenciées, l'une de proximité, entre disciplines voisines, l'autre appelée «dédaléenne », plus affranchie, plus ouverte. Il faut enfin insister sur le manque de légitimité dont peuvent souffrir de jeunes chercheurs face à des institutions d'enseignement et de recherche (encore?) fortement structurées autour des disciplines.

\section{Keywords:} interdisciplinarity, young researchers, epistemology, scientific legitimacy

\begin{abstract}
Young researchers and interdisciplinarity in social sciences. Reconsidering practices. Individual practice of interdisciplinarity by young researchers is rarely called into question. Yet it is quite specific. Borrowing concepts, methods, and theories from other disciplines is motivated by intellectual curiosity and by a desire to improve the research object and framework. The initial course is, however, often intuitive and follows the researcher's affinity. The temptation to forge onwards, to achieve exhaustiveness, the lack of appropriate methodological background are all very real risks. It is thus important that young researchers consider scientific requirements within the original frame of interdisciplinarity. This is all the more necessary in view of pressing societal expectations toward the researcher, notably as an expert or a specialist. The difficulty, then, is to achieve epistemological coherency and in order to do so, acquire crucially important "multicompetence". Interdisciplinarity is indeed plural. Two interdisciplinarity courses may be distinguished: one based on proximity between two neighbouring disciplines, the other freer and more open, termed "dédaléenne" in reference to Deadalus's maze. Last, we wish to insist on the lack of legitimacy from which young researchers may suffer confronted as they are by research and education institutions which are strongly structured around disciplines. This should cause us to reflect upon the increasingly eclectic initial training programmes of young researchers who nowadays question their own interdisciplinarity.
\end{abstract}

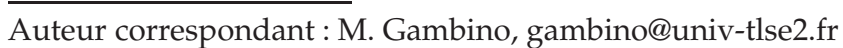




\section{Introduction}

Nous suivons avec intérêt les contributions régulières au forum interdisciplinarité de cette revue et avons souhaité y proposer un regard qui, nous semble-t-il, n'a été que peu abordé jusqu'alors. En effet, notre objectif est de réfléchir aux pratiques interdisciplinaires ${ }^{1}$ individuelles - alors qu'elles sont généralement abordées en tant que démarches collectives - et de mettre en évidence les enjeux que ces questions recouvrent pour les jeunes chercheurs en sciences sociales que nous sommes. Si la pratique individuelle de l'interdisciplinarité soulève déjà en soi un ensemble de questionnements, il nous a semblé que le jeune chercheur était confronté à des problématiques particulières, directement liées à son statut. Par l'individualisation de l'interdisciplinarité, la pratique passe d'un enjeu collectif à un enjeu épistémologique, conduisant à s'interroger sur le fondement et la nature des sciences.

Pratiquer l'interdisciplinarité n'est pas chose aisée, et elle laisse souvent un sentiment de malaise chez le jeune chercheur. Ce sentiment provient à la fois d'un inconfort personnel à se placer dans une démarche dont on connaît encore mal les tenants et les aboutissants, et de mises en garde régulières, plus ou moins expresses, provenant d'enseignants-chercheurs confirmés. Nous sommes alors régulièrement sommés de justifier notre positionnement, d'expliciter notre démarche. Par ailleurs, si l'interdisciplinarité constitue une épreuve parfois déplaisante pour des apprentis chercheurs, c'est aussi en partie parce qu'elle donne l'impression d'une impossible spécialisation : elle laisse un goût d'inachevé, conforté par le sentiment de ne rien maîtriser et de faire dans l'approximatif. Ce sentiment est renforcé par l'image de «touche à tout » qui est nous est alors renvoyée.

C'est pour questionner nos propres pratiques et mettre en lumière les problèmes auxquels les jeunes chercheurs interdisciplinaires doivent faire face que nous avons souhaité fournir ici un effort de réflexion. Nous explorerons ainsi les justifications, les raisons qui poussent à se positionner dans l'interdisciplinarité et la façon dont on peut mener à bien une telle démarche, sans oublier de présenter les errances et les difficultés auxquelles le jeune chercheur peut être confronté, que ce soit en termes de légitimation de sa démarche (à ses propres yeux ainsi qu'à ceux de la communauté scientifique) que de positionnement personnel dans les institutions.

\footnotetext{
1 Nous entendrons l'interdisciplinarité dans son sens le plus large, c'est-à-dire le fait d'avoir recours à des disciplines différentes, que ce soit dans une optique de complémentarité ou d'emprunt des éléments disciplinaires (théories, concepts, méthodes). En outre, nous utiliserons pluridisciplinarité pour désigner une simple juxtaposition ou accumulation d'apports relevant de différentes disciplines.
}

\section{De l'objet de recherche à la mobilisation de plusieurs disciplines}

Comment en vient-on à inscrire sa recherche dans une perspective interdisciplinaire? Dans plusieurs écrits sur l'interdisciplinarité, l'idée selon laquelle c'est la complexité de certains objets d'étude (Boiffin, 2004; Deffontaines et Hubert, 2004) - tels l'environnement, le territoire, le climat ou encore le développement - qui appelle la pratique interdisciplinaire est récurrente. En effet, la nature desdits objets est conçue comme un système dynamique, "irréductible à une seule dimension » (Klein, 2004). L'accumulation des savoirs et le croisement des regards, les interactions entre phénomènes, caractérisent de façon indéniable les objets de recherche : la complexité est devenue une valeur intrinsèque de la connaissance et de la recherche en général. Cette tendance est sans doute directement liée à l'essor et au succès des théories constructivistes, qui ont amené à l'obsolescence de nombreuses approches normatives. Ce faisant, elles ouvrent le champ des corpus scientifiques, tout en conférant au savoir un caractère relatif et potentiellement incomplet. L'irruption de la systémique, autour de chercheurs comme J.-L. Le Moigne ou E. Morin, en est un exemple caractéristique.

Toutefois, nous pensons que ce n'est pas seulement la complexité des objets de recherche, mais également - et dans notre cas surtout - la façon dont l'objet est problématisé qui nous pousse à mobiliser l'interdisciplinarité. Nous sortons de notre discipline pour rechercher d'autres éléments de problématisation, d'explication, ou parce que nos outils théoriques et méthodologiques semblent restreindre notre raisonnement. Il s'agit donc d'ouvrir les perspectives de la recherche, d'étoffer les moyens d'y répondre et d'aiguiser le sens critique. Notre travail de chercheur complexifie le questionnement, et ce d'autant plus quand l'enseignement reçu nous a appris à observer un même phénomène sous différents angles. Cela se passe tant sur le plan des théories (la géographie devient sociale ${ }^{2}$ 'économie devient cognitive, la psychologie devient sociale ${ }^{3}$ ) que sur celui des méthodes.

Par ailleurs, la démarche interdisciplinaire répond aussi à des impératifs liés aux évolutions de la société et de la place qu'y occupe la recherche. La fonction du chercheur a changé (Pestre, 2004). Dans son travail, il est ainsi appelé tantôt à donner un avis d'expert sur une question complexe, tantôt à mettre son savoir de spécialiste en relation avec d'autres savoirs spécialisés. Tout à la fois scientifique, trouveur, expert, le chercheur doit être capable de tout comprendre, de tout expliquer et de tout résoudre. Pour répondre à ces évolutions, les compétences doivent

\footnotetext{
${ }^{2}$ Dans un contexte de réinterprétation des théories, la géographie, élargit ses références à des modèles d'analyses empruntés aux sciences sociales (Bailly, 1991).

${ }^{3}$ Sur la psychologie, voir Valade, 1999 : pp. 13-15.
} 
se diversifier, la connaissance se doit d'être précise tout en relevant de plusieurs domaines. Il nous semble alors nécessaire d'avoir, au moins, une attitude d'ouverture à l'égard des marges disciplinaires. Il faut avoir au mieux une connaissance épistémologique et une culture générale approfondie, de façon à connaître les possibilités d'allier et de compiler diverses avancées théoriques pour les orienter vers la résolution d'un problème empirique (dont il faut également connaître, au moins en surface, les multiples facettes). En d'autres termes, il faut avoir acquis ce qu'E. Morin (1990) qualifie de "polycompétence du chercheur». Ces diverses figures qui nous sont renvoyées par les acteurs de terrain, les élus, mais aussi par la société dans son ensemble nous mènent à l'interdisciplinarité et font que cette dernière se présente davantage comme une stratégie que comme une véritable approche.

Mais, derrière ces évolutions scientifiques et sociétales amenant à l'interdisciplinarité, le chercheur est luimême souvent mû par des motivations d'un autre type : les pratiques individuelles répondent à un dessein plus ou moins assumé d'exhaustivité et de liberté. Nous nous expliquons. La tentation est grande pour le chercheur de mener à bien une recherche avec l'ambition de saisir «l'universel-concret» (Mathieu, 1992). Il cherche alors les interactions, les interrelations en œuvre concernant l'objet qu'il observe/questionne, et choisit l'interdisciplinarité comme un moyen pour atteindre ces objectifs. Bien souvent, le jeune chercheur est d'abord motivé par un appétit de savoir et une tentation de toucher à tout, et son positionnement se complexifie avec l'avancement de ses lectures. Plus ces dernières sont diversifiées, plus les facettes éclairant un même objet se multiplient et plus l'appétit de savoir, tout comme la volonté d'être exhaustif, s'exacerbent.

Quant à l'aspect libérateur de l'interdisciplinarité, parfois vanté par les chantres de ce type de pratiques, il tient essentiellement au fait que le chercheur, en chaussant de multiples "lunettes", sort de ce que S. Charbonneau (2005) qualifie «d'enfermement disciplinaire ». En recourant à l'interdisciplinarité, nous nous affranchissons de certaines normes, puisqu'il n'existe ni méthodes ni procédures communément reconnues pour mener à bien une telle démarche. L'interdisciplinarité est ainsi créative en soi, à la fois parce qu'elle ouvre des potentialités et parce que, faute de méthodes reconnues, le chercheur se doit d'inventer la façon dont vont s'articuler et se nourrir les différents apports (extra)disciplinaires qu'il mobilise.

\section{De la pratique intuitive à la démarche scientifique}

Nous avons exploré plus haut les motivations qui expliquent le choix d'une démarche interdisciplinaire.
Mais, dans les faits, nous y entrons la plupart du temps par des chemins moins volontaristes, moins conscients, qui, s'ils sont indirectement liés à des enjeux sociétaux ou à des ambitions inavouées, ne sont pas encore perçus comme conduisant à une démarche singulière. Le jeune chercheur, en particulier, commence souvent à faire de l'interdisciplinarité de façon non intentionnelle, par curiosité intellectuelle ou par tâtonnement.

Sa démarche première se fonde alors pour une large part sur l'intuition, que R. Franck (1999) définit comme l'aperception immédiate, globale et non formalisée de l'objet lui-même. Il synthétise cette connaissance tacite et subjective sous le terme de «connaissances ordinaires ». L'intuition prend dès lors une part très importante dans la construction de la réflexion, puisqu'elle est utilisée pour mobiliser des champs de savoir différents, dont on a l'intuition qu'ils concourent à éclairer un objet de recherche. C'est elle qui permet de faire appel à des savoirs discrets et sans relation apparente; elle nous amène à lier des éléments de connaissances qui sont en nous, mais que les postures disciplinaires n'appellent pas à mettre en perspective. En d'autres termes, l'intuition ne s'encombre pas de déterminer les catégories de savoir qu'elle mobilise et nous oriente vers une connaissance «a-disciplinaire ${ }^{4}$ » et immédiate des propriétés d'un système complexe. Audelà de cette possibilité ouverte de s'extraire des disciplines lors de la première confrontation avec un objet de recherche, l'intuition permet aussi une certaine porosité entre la vie sociale du chercheur et son entendement du monde ${ }^{5}$. Elle lui permet de faire appel à des expériences quotidiennes, à des inspirations multiples qui ne relèvent pas à proprement parler du champ scientifique. Ainsi, une discussion, un film ou encore un événement d'actualité pourront créer un « déclic » dans l'avancement de la recherche, qu'une démarche strictement méthodologique n'aurait pu susciter.

Si l'intuition joue un rôle très important dans toute démarche de construction du savoir, qu'elle se revendique ou non de l'interdisciplinarité, il nous semble particulièrement important de souligner son apport dans ce cas précis, du fait, entre autres, de l'absence d'une méthodologie. Le chercheur se trouve en effet face à un vaste ensemble de possibles et l'intuition guide alors sa recherche. Elle l'aidera à avoir une conscience « ordinaire » du chemin qui pourrait être emprunté, de sa validité et de sa légitimité immédiate. Bien sûr, il ne s'agit pas de donner à l'intuition une valeur scientifique en tant que justification nécessaire et suffisante d'une posture de recherche, mais bien de lui reconnaître un rôle indispensable et fondateur pour l'avancement de la recherche. Elle ne peut prétendre être un aboutissement; elle est bien plus une

\footnotetext{
${ }^{4}$ C'est-à-dire qui n'est pas positionnée dans des disciplines.

5 Sur ces interactions entre les connaissances quotidiennes (émanant de la vie sociale) et le savoir "savant", voir H. Nowotny (2003), qui parle de savoir transgressif.
} 
amorce et un guide. On ne peut, dans ce cadre, lui accorder une validité scientifique que pour autant qu'elle soit suivie d'une démarche rigoureuse et méthodique.

La première des nécessités pour réintégrer des tâtonnements et des intuitions dans une démonstration scientifique est de prendre conscience de la nature interdisciplinaire de sa posture. Autrement dit, il faut reconnaître le caractère innovant et particulier de son cheminement épistémologique pour pouvoir ensuite l'aborder comme tel et savoir qu'il aura besoin d'être éprouvé et légitimé. Trop souvent, le recours à d'autres disciplines se fait inconsciemment, de sorte que tout le questionnement autour de la démarche elle-même est occulté du travail, qui ne retiendra que les résultats obtenus. Tout scientifique, mais particulièrement les jeunes chercheurs que nous sommes, se doit d'avoir conscience des chemins qu'il emprunte; ceci, non seulement pour questionner et objectiver une démarche, mais aussi pour prendre conscience d'un positionnement individuel au sein d'un contexte scientifique et institutionnel.

Cette étape étant accomplie, il existe encore de nombreux obstacles à surmonter avant de produire un travail cohérent et pertinent, qui ait su construire un raisonnement valide à partir d'apports disciplinaires éclectiques. L'une des phases particulièrement critiques de la recherche est en effet celle de l'articulation d'apports différents dans une même démonstration. Ce temps de la recherche requiert une attention particulière à deux égards : d'une part, les éléments de la démonstration doivent être tous orientés et articulés vers la résolution d'une même problématique (ils sont donc nécessairement inscrits dans une démonstration unique et autonome); d'autre part, cette démonstration doit être épistémologiquement cohérente, ce qui suppose une bonne connaissance des disciplines mobilisées et de leur fonctionnement. C'est sans doute ici que réside l'une des principales difficultés liées à l'interdisciplinarité, et que se repose la question de la «polycompétence » du chercheur. Il lui faut en effet avoir des connaissances à la fois variées et approfondies, ce qui n'est pas sans poser problème, en particulier pour le jeune chercheur.

Dans tous les cas, lorsque la démarche interdisciplinaire est effectuée de façon consciente et pleinement assumée, cela conduit à s'interroger sur ses pratiques et, partant, sur la science que l'on produit. Il s'ensuit nécessairement un questionnement sur l'interdisciplinarité elle-même, sur ses formes et ses caractéristiques ; car, assurément, il n'y a pas une, mais des interdisciplinarités.

\section{Quelles interdisciplinarités, quelles pratiques?}

Alors qu'une démarche collective, au sein de programmes de recherche, par exemple, peut permettre de résoudre certains problèmes de compétences méthodologiques ou théoriques, a-t-on individuellement suffisamment d'envergure ou d'expérience dans différentes disciplines pour relever les défis de l'interdisciplinarité? Pour répondre à cette question, nous avons identifié diverses façons de mener individuellement une recherche interdisciplinaire et en avons soulevé les enjeux qui peuvent être problématiques pour de jeunes chercheurs.

Deux pratiques peuvent être distinguées. L'une, couramment observée autour de nous et décrite par certains, est de faire de l'interdisciplinarité de proximité (Struppa, 2002; Jollivet et Legay, 2005). Cette pratique consiste à utiliser des concepts, des théories et des méthodes de disciplines proches de la sienne, donc de profiter du voisinage disciplinaire. L'objectif est de pouvoir proposer des interprétations plus riches, originales, du processus étudié. Le chercheur se tourne d'ailleurs souvent vers des disciplines et des outils qu'il estime facilement mâ̂trisables, vers lesquels le portent ses compétences déjà acquises et avec lesquels il a des affinités. L'interdisciplinarité autorise un décentrage, une perspective de biais, qui permet de se donner d'autres modes de raisonnement, d'autres catégories de pensée, de diversifier les modes de compréhension. L'interdisciplinarité de proximité peut s'effectuer de deux façons. Dans certains cas, elle peut être momentanée, les emprunts à d'autres disciplines pouvant s'avérer commodes pour utiliser des théories, des concepts, des méthodes élaborés par d'autres, sans les redéfinir ni les adapter. Cette pratique joue sur la porosité des frontières disciplinaires, mais le chercheur continue alors à définir son objet de recherche et son interrogation de façon disciplinaire. Dans d'autres cas, ces emprunts peuvent être davantage appropriés par le chercheur. L'interdisciplinarité relève ici du transfert, de la traduction de méthodes ou de théories explicatives, puisque nombreux sont les chercheurs qui adaptent, interprètent et redéfinissent ce qu'ils sont allés chercher.

Une autre pratique de l'interdisciplinarité, que l'on pourrait qualifier de "dédaléenne », consiste à vouloir comprendre comment un objet de recherche peut être questionné par d'autres disciplines. C'est l'expérience de jeunes chercheurs qui se trouvent limités dans la compréhension de leur objet et qui se tournent vers de nouvelles disciplines pour trouver des éléments de compréhension et d'analyse plus heuristiques. Dès lors, la recherche progresse grâce à la mise en œuvre de concepts opératoires théorisés et explorés par une autre discipline. C'est tout le questionnement de départ qui s'en trouve chamboulé et déplacé vers des disciplines distinctes. Ici, les disciplines se trouvent véritablement rapprochées parce que des théories, des concepts ou des méthodes circulent au sein d'une même recherche et, partant, se trouvent repensés. Ce qui au départ relevait de l'emprunt à une autre discipline aboutit à une fusion des deux disciplines. Toutes deux sont utiles et heuristiques, d'importance égale dans 
le questionnement, à tel point qu'il est parfois possible de ne plus savoir laquelle nourrit l'autre. Il s'ensuit une difficulté pour le chercheur, qui, constamment tiraillé entre deux positionnements disciplinaires, ne sait lequel revendiquer.

Au niveau individuel, les pratiques de l'interdisciplinarité peuvent donc être plurielles ${ }^{6}$. Cette présentation est une réduction de la réalité complexe de notre travail, mais il nous semble utile de distinguer ces deux formes de pratiques interdisciplinaires que nous venons de distinguer pour mettre en avant les difficultés que nous sommes susceptibles de rencontrer.

Dans un cas, le jeune chercheur pratique une interdisciplinarité très limitée, bien en deçà de ses ambitions. Sa pratique est instrumentale et incomplète, elle lui pose problème du fait d'un manque de recul et de connaissances épistémologiques suffisantes. Il court le risque de vouloir tout comprendre, tout connaître sur une petite partie du «savoir » qu'il tente de maîtriser, et de produire une recherche trop complexe, mal appropriée et parfois difficilement compréhensible. Cette visée exhaustive représente aussi un écueil dissimulé de l'interdisciplinarité : une fuite en avant qui permet au jeune chercheur de se perdre dans ses investigations, dans son travail théorique et de retarder, de manière délibérée ou non, les étapes de la recherche qu'il appréhende. Soulignons aussi que, sous couvert d'un impératif scientifique, l'interdisciplinarité est un moyen de suivre une voie indépendante et centrée sur des intérêts propres. De la sorte, derrière une démarche innovante et créative, se cache parfois l'égocentrisme de celui qui la pratique, qui l'utilise pour aborder des thématiques qui lui sont chères, concourant finalement à un autre type d'enfermement, centré celui-ci sur des intérêts personnels. En bref, cette pratique qui vise la complémentarité des disciplines fait apparaître des «spécialités mixtes » (Valade, 1999) : un savoir basé sur le dialogue entre les disciplines, sur l'hybridation des outils et des concepts mis en œuvre. Ces "spécialités mixtes » permettent aux chercheurs de se démarquer et de trouver une singularité pour ancrer et identifier leurs recherches. Elles permettent par ailleurs de voir que les disciplines ont une tendance fédérative et se nourrissent de la porosité de leurs frontières en laissant se créer de nouveaux domaines d'action et de création scientifique.

Dans l'autre cas où plusieurs disciplines fusionnent, en tant que doctorantes, il semble que notre pratique de l'interdisciplinarité soit un exercice de style, car elle doit rentrer dans des codes disciplinaires. Dans ce contexte, elle a pour but à la fois de valoriser nos compétences dans une discipline et de montrer ce que l'on peut y apporter. Il est certes possible de faire des emprunts pour favori-

\footnotetext{
${ }^{6}$ Pour une référence à des travaux de typologie sur les pratiques interdisciplinaires, voir E. Morin (1990) ou encore D. Struppa (2002).
}

ser une lecture complexe de l'objet de recherche, comme complément d'analyse, mais il faut pouvoir justifier auprès de sa discipline de « rattachement » de tels transferts et de tels détours. Utiliser un vocabulaire ou approfondir un objet sous des questionnements d'autres disciplines induit en effet un problème de positionnement. Finalement, le jeune chercheur a le sentiment que la pratique de l'interdisciplinarité est contradictoire avec une quelconque spécialisation (dans sa propre discipline) et il peut se trouver démuni face à la question de son statut: de quoi est-il spécialiste, d'une discipline, d'un terrain, d'un objet, de méthodes particulières? Lorsque le cheminement de la recherche progresse par des choix qui amènent à se pencher sur des objets de plus en plus éloignés de sa discipline, que les méthodes sont empruntées à d'autres, comment affirmer et légitimer son appartenance à la discipline de départ? S'il se spécialise, c'est à la marge de sa discipline.

Toutes ces questions résonnent de manière particulière quand le jeune chercheur se retrouve en situation d'évaluation, notamment face aux institutions d'enseignement et de recherche.

\section{La difficile reconnaissance institutionnelle}

On le sait, les institutions d'enseignement et de recherche sont structurées autour des disciplines (Faure, 1992). La reconnaissance institutionnelle peut donc constituer une réelle difficulté pour le jeune chercheur interdisciplinaire. Les systèmes d'évaluation, notamment lors des recrutements, fonctionnent sur des bases disciplinaires. Les universités sont probablement les plus soucieuses de recruter des jeunes chercheurs monodisciplinaires puisque, précisément, elles leur confieront l'enseignement d'une discipline. Il est aisément compréhensible que les principes d'évaluation qui ont, pendant des décennies, reposé sur des fondements disciplinaires ${ }^{7}$ ne puissent être adaptés que progressivement à l'interdisciplinarité. Si, par conséquent, de grands organismes de recherche - le CNRS, par exemple - sont aujourd'hui en mesure d'évaluer des individus qui ont des parcours pluridisciplinaires et qui proposent des travaux pluridisciplinaires, c'est à certaines conditions.

Pour la plupart des institutions, l'interdisciplinarité n'est concevable que si elle ne remet pas en cause les fondements des disciplines, mieux, si elle conforte les disciplines en place : "[la] pratique de la transdisciplinarité exige, au contraire, le renforcement constant du «noyau dur » [des différentes disciplines] » (CNRS, 2002, p. 13). Les instances évaluatrices admettront bien plus

\footnotetext{
${ }^{7}$ Même si les raisons de cette inertie ne sont pas toujours avouables : rapports de pouvoir entre disciplines et au sein de celles-ci.
} 
l'incursion dans une discipline connexe d'un jeune chercheur facilement identifiable au sein de sa discipline que celle d'un jeune chercheur qui se situe dans un champ nouveau et/ou marginal de sa propre discipline. Dans le premier cas, on se félicitera et le félicitera de sa curiosité, voire de sa prise de risque, on excusera ses approximations, puisqu'il a fait ses preuves dans sa discipline. Dans le second cas, on se méfiera et on se moquera de ses incartades prétentieuses, on pressentira peut-être une « instabilité intellectuelle »; c'est sûr, il aurait mieux valu qu'il s'en tienne aux acquis de sa discipline.

Questionner l'évaluation de l'interdisciplinarité nécessiterait de questionner plus globalement les principes de l'évaluation de la recherche elle-même : comment dépasser des critères quantitatifs et intégrer des repères épistémologiques (curiosité et culture scientifiques, originalité des problématiques et des interprétations proposées) ? Plus généralement encore, quelles sont les attentes, aujourd'hui, à l'égard d'un travail scientifique en sciences humaines et sociales? Mais il conviendrait surtout, pour nous, de clarifier les exigences à l'égard d'une recherche effectuée notamment par les doctorants. Les jeunes chercheurs doivent-ils démontrer qu'ils sont capables de construire une recherche et de répondre à une question ou qu'ils ont acquis les bases nécessaires à la maîtrise d'une discipline? Faut-il imposer qu'ils maîtrisent les «fondements » d'une discipline? Quels fondements? Définis par qui? Définis comment? Doiton l'imposer à ceux qui défrichent de nouveaux objets, qui construisent des problématiques originales? Peut-on l'imposer étant donné les conditions actuelles de production des thèses (durée raccourcie, faible nombre de bourses, etc.)?

Des chercheurs l'ont déjà fait remarquer - dans des numéros précédents de NSS, notamment Deffontaines et Hubert (2004) -, les exhortations à l'interdisciplinarité sont bien trompeuses : encouragée en amont, l'interdisciplinarité est vilipendée en aval du processus de recherche. Mais peu nombreux sont ceux qui envisagent la grande responsabilité à l'égard des jeunes chercheurs : "N'envoyons-nous pas nos thésards au "casse-pipe" en les entraînant sur les chemins périlleux de l'interdisciplinarité ? [...] Trouveront-ils des postes s'ils n'ont pas un profil clairement disciplinaire? » (Joulian et al. 2005, p. 288).

Ce sont là des points trop peu abordés au long des cursus universitaires. Les formations initiales du supérieur de plus en plus éclectiques (systèmes d'options «à la carte »), s'ajoutant à des enseignements volontairement interdisciplinaires (tels la filière AES (Administration économique et sociale), les écoles d'ingénieurs ou certains mastères), ouvrent l'étudiant à diverses disciplines, mais ne forment pas à la pratique interdisciplinaire. Pire, la nature des disciplines, leur délimitation, leur teneur, sont la plupart du temps absentes de l'enseignement, qui se concentre sur leur contenu. Les enseignements sont le plus souvent une compilation, voire une superposition de regards différents, connectés au mieux par des thématiques communes, que le jeune chercheur devra apprendre à classer, structurer et utiliser, dans des environnements qui sont en retour souvent organisés autour des disciplines ${ }^{8}$. Nous ne voyons pas là une volonté délibérée de laisser le jeune chercheur trouver sa place dans son environnement disciplinaire ; bien au contraire, c'est souvent parce que l'enseignant lui-même ne détient pas de " recette interdisciplinaire », de méthode pour guider le jeune chercheur dans son travail et dans son positionnement que l'on se trouve dans une telle situation. Cela est sans doute à lier à la propre formation des enseignants, qui eux-mêmes n'ont pas été préparés pour faire face à cette évolution.

En conclusion, le jeune chercheur peut se trouver devant une multitude de questionnements, voire d'errances, lors de son parcours : comment connaître les enjeux liés à l'interdisciplinarité et quelles sont les aptitudes dont on doit faire preuve pour l'aborder? Il est fort regrettable que le jeune chercheur se retrouve parfois si seul face à ces importantes questions épistémologiques et face aux diverses instances d'évaluation. Bien souvent, l'interdisciplinarité repose sur des expériences vécues individuellement, sur lesquelles l'échange n'est pas formalisé, et que l'on ne parvient pas encore à déconstruire et à croiser. On ne peut que louer l'entreprise salutaire de NSS. Il ne faut pas douter de l'interdisciplinarité : certains chercheurs ont été notoirement à cheval sur plusieurs disciplines, ce qui ne les a pas empêchés de produire un travail d'excellence, largement reconnu et revendiqué par l'ensemble des disciplines auxquelles ils ont su faire des emprunts. Ainsi, F. Braudel, M. Foucault, M. Serres ou encore E. Morin nous montrent qu'il est possible de faire de la «bonne interdisciplinarité ».

\section{Références}

Bailly, A., 1991. Les concepts de la géographie humaine, Paris, Masson.

Boiffin, J., 2004. Territoire : agronomie, géographie, écologie, où en est-on? Le point de vue d'un chercheur agronome, Natures Sciences Sociétés, 12, 3, 307-309.

Charbonneau, S., 2005. Réflexion d'un juriste sur l'enfermement disciplinaire, Natures Sciences Sociétés, 13, 1, 58-61.

CNRS, 2002. Projet d'établissement du CNRS, Paris, CNRS.

\footnotetext{
${ }^{8}$ Nous ne remettons pas ici en cause la pertinence et la nécessité de proposer des formations multidisciplinaires. Bien au contraire, nous sommes convaincues de leur richesse, de leur utilité et de l'extraordinaire éventail de possibilités qu'elles ouvrent à tout étudiant. Ayant été formées sous le signe de la multidisciplinarité, notre vécu nous amène à en reconnaître toute la valeur, tout en en percevant certaines limites.
} 
Deffontaines, J.-P., Hubert, B., 2004. Un regard sur l'interdisciplinarité à l'Inra. Point de vue de deux chercheurs du département Sciences pour l'action et le développement (SAD), Natures Sciences Sociétés, 12, 2, 186-190.

Faure, G.O., 1992. La mise en œuvre de l'interdisciplinarité : barrières institutionnelles et intellectuelles, in Portella, E. (Ed.), Entre savoirs. L'interdisciplinarité en acte : enjeux, obstacles, perspectives, Toulouse, Erès, 109-116.

Franck, R., 1999. La pluralité des disciplines, l'unité du savoir et les connaissances ordinaires, Sociologie et sociétés, 31, 1, 129-142.

Jollivet, M., Legay J.-M., 2005. Canevas pour une réflexion sur une interdisciplinarité entre sciences de la nature et sciences sociales, Natures Sciences Sociétés, 13, 2, 184-188.

Joulian, F., de Cheveigné, S., Le Marec, J., 2005. Évaluer les pratiques interdisciplinaires, Natures Sciences Sociétés, 13, 3, 284-290.

Klein, J., 2004. Interdisciplinarity and complexity: an evolving relationship, $E: C O, 6,1-2,2-10$.
Mathieu, N., 1992. Géographie et interdisciplinarité : rapport naturel ou rapport interdit?, in Jollivet, M. (Ed.), Science de la nature, science de la société : les passeurs de frontières, Paris, CNRS Éditions, 129-154.

Morin, E., 1990. Sur l'interdisciplinarité, in Kourilsky, F. (Ed.), Actes du colloque Carrefour des sciences : session plénière du Comité national de la recherche scientifique : l'interdisciplinarité, 12 et 13 février 1990, Palais de L'Unesco/CNRS, [s.1.], PAPCOM.

Nowotny, H., 2003. Le potentiel de la transdisciplinarité, http ://www.interdisciplines.org/interdisciplinarity/ papers/5/language/fr (consulté en décembre 2005).

Pestre, D., 2004. L'évolution des champs de savoir, interdisciplinarité et noyaux durs. Communication au Conseil scientifique du CNRS (14 janvier 2002), Natures Sciences Sociétés, $12,2,191-196$.

Struppa, D., 2002. The nature of interdisciplinarity, The Journal of the Association for General and Liberal Studies, 30, 1, 97-105.

Valade, B., 1999. Le «sujet» de l'interdisciplinarité, Sociologie et sociétés, 31, 1, 11-21. 